\title{
Estudio de muertes por causas violentas: un análisis de tendencia en jóvenes, Argentina, 2000-2008
}

\section{Study about violent deaths: an analysis of trends for youth in Argentina, 2000-2008}

\author{
María Soledad Burrone¹, Mónica Bella², Laura Acosta³, Belén Villace ${ }^{4}$, \\ María Josefina López de Neira ${ }^{5}$, Ruth Fernández ${ }^{6}$, Julio Enrique Enders ${ }^{7}$
}

\begin{abstract}
Resumen
Introducción: Las muertes de jóvenes por causas violentas son las principales causas de muerte en Latinoamérica. En función de la crisis económico-social producida en Argentina a partir del 2001, el objetivo del presente trabajo fue analizar la tendencia de mortalidad por causas violentas en jóvenes de 10 a 24 años en el período 2000-2008. Metodología: Se utilizaron las Bases de Datos de los Informes Estadísticos de Defunción y los Anuarios de Estadísticas Vitales. Fueron calculadas tasas de mortalidad por causas violentas por los 100.000 habitantes, utilizando la clasificación de la Clasificación Estadística Internacional de Enfermedades y Problemas Relacionados con la Salud (CIE-10). El análisis estadístico se llevó a cabo teniendo en cuenta la naturaleza de la variable. Resultados: La tasa de mortalidad por causas violentas en el 2000 fue de $42,48 \%$ muertes, y en el 2008 fue de 43,09\% $\%_{000}$, con una mayor incidencia de muerte por causas violentas en varones $(p=0,0015)$. Dentro de las causas violentas se observó un incremento en los accidentes de tránsito en ambos sexos y agresiones predominantemente en varones. La mayor tasa de suicidio ocurrió durante el periodo 2000-2005. Conclusiones: Teniendo en cuenta que todas son causas de muertes evitables, es imperiosa la reflexión de políticas públicas de salud.
\end{abstract}

Palabras clave: violencia; tasa de mortalidad; adolescente.

\begin{abstract}
Introduction: Violence is the leading cause of death among young people in Latin America. The objective of this study was to analyze the trends of youth (ages 10 to 24) mortality resulting from violence, over the period 2000-2008, in the context of the socioeconomic crisis that started in Argentina, in 2001. Methodology: Databases of the Statistical Reports of Death and Vital Statistics Yearbooks were used. Violent deaths were defined by the International Statistical Classification of Diseases and Related Health Problems (ICD-10), and violent mortality rates for every 100,000 inhabitants were calculated. Statistical analysis was performed according to the nature of the variable involved. Results: Violent mortality rates were $42.48 \%$ o000 deaths for 2000 , and
\end{abstract}

Trabajo realizado en la Escuela de Salud Pública, Facultad de Ciencias Médicas de la Universidad Nacional de Córdoba - Córdoba, Argentina. 'Médica, Magister en Salud Pública en la Escuela de Salud Pública, Facultad de Ciencias Médicas, Universidad Nacional de Córdoba - Córdoba, Argentina. 2Doctora en Medicina y Cirugía; Especialista en Psiquiatría en la Escuela de Salud Pública, Facultad de Ciencias Médicas, Universidad Nacional de Córdoba Córdoba, Argentina.

${ }^{3}$ Licenciada en Nutrición; Becaria del Consejo Nacional de Investigación Científica y Tecnológica (CONICET); Profesora Asistente en la Escuela de Salud Pública, Facultad de Ciencias Médicas, Universidad Nacional de Córdoba - Córdoba, Argentina.

${ }^{4}$ Licenciada en Nutrición; Becaria en la Secretaría de Ciencia y Tecnología de la Universidad Nacional de Córdoba (SECyT); Maestranda en Salud Pública en la Escuela de Salud Pública, Facultad de Ciencias Médicas, Universidad Nacional de Córdoba - Córdoba, Argentina.

${ }^{5}$ Diplomada en Salud Pública; Especialista en Pediatría; Asesora de la Organización Panamericana de Salud (OPS), Profesor Asistente en la Escuela de Salud Pública, Facultad de Ciencias Médicas, Universidad Nacional de Córdoba - Córdoba, Argentina.

${ }^{6}$ Doctora en Medicina y Cirugía; Magister en Docencia; Profesora Titular en la Escuela de Salud Pública, Facultad de Ciencias Médicas, Universidad Nacional de Córdoba - Córdoba, Argentina.

Doctor en Ciencias Químicas; Profesor Titular Plenario en la Facultad de Ciencias Médicas, Universidad Nacional de Córdoba - Córdoba, Argentina. Dirección para correspondencia: Ruth Fernández - Facultad de Ciencias Médicas - Universidad Nacional de Córdoba - Escuela de Salud Pública - Enrique Barros, s/n - Ciudad Universitaria - Córdoba, Argentina - E-mail: aruthfernandez@gmail.com

Soporto financiero: Fondo Nacional de Ciencia y Tecnología (FONCyT), Argentina. Las bases de datos fueron suministradas por la Dirección de Estadísticas e Información de Salud, Argentina.

Conflicto de interese: nada a declarar. 
$43.09 \%$ for 2008 , with the incidence being higher for males $(\mathrm{p}=0.0015)$. For both sexes, there was an increase of violent deaths due to car accidents. There was also an increase of deaths by aggression in males. The highest suicide rate corresponded to the period 2000-2005. Conclusions: Since these violent deaths are preventable, reflection on public health policies is urgent.

Keywords: violence; mortality rate; adolescent.

\section{INTRODUCCIÓN}

La mortalidad por causas violentas abarca las lesiones no intencionales, que comprenden los accidentes, y las intencionales. Éstas últimas pueden ser lesiones autoinfligidas intencionalmente o causadas por terceros (agresiones) ${ }^{1}$. La mortalidad por causas violentas ha sido analizada en la población general y en particular en menores de 24 años, en estudios previos que abordaron la información registrada en la región de las Américas ${ }^{2,3}$. Así, las muertes violentas en adolescentes y jóvenes es uno de los problemas sanitarios más importantes constituyendo la principal causa de muerte en el grupo $^{4-8}$. Entre las muertes por causas violentas se identifica a los accidentes, el suicido, y el homicidio.

Las estimaciones efectuadas por la Organización Mundial de Salud (OMS) refieren que en la actualidad mueren por causas violentas 4,5 millones de personas y se suicidan un millón en el mundo y esta cifra ascendería a 1,5 millones para $2020^{8,9-11}$. En Argentina, la mortalidad por suicidio en adolescentes y jóvenes se incrementó de 1,5 cada 100.000 habitantes en 1990 a 6,1 cada 100.000 habitantes en 2000, mostrando además que las edades con mayor incremento estaban comprendidas entre los 20 y 25 años $^{7,11}$.

Se conoce que la muerte por causas violentas se constituye en un problema de salud pública de complejidad respondiendo a factores de ordenes biológico, psicológico, y social, afectando a la población más joven lo que lleva a ocasionar pérdidas afectivas y económicas. Las muertes por causa violenta son posibles de prevenir siempre que se cuente con información con respecto a los factores que intervienen en ellas y se desarrollen programas de prevención y tratamiento oportunos.

A partir de lo expuesto y en función de la crisis económica social producida en Argentina a partir del año 2001, el objetivo del presente trabajo fue analizar la tendencia de mortalidad por causas violentas en Argentina en adolescentes y jóvenes de 10 a 24 años en el período 2000-2008, analizando en particular la muerte por suicidio en este grupo, que tal como se expresara registra antecedentes de incremento desde la década del 1990.

\section{MATERIALES Y MÉTODOS}

Las defunciones registradas en adolescentes y jóvenes de 10 a 24 años de edad de ambos sexos, en el período 2000 a
2008 en Argentina, fueron obtenidas a partir de la Base de Datos de los informes estadísticos de defunción (IED), proporcionados por la Dirección de Estadística e Información de Salud del Ministerio de Salud de la Nación, que es la institución responsable de las estadísticas oficiales de salud en Argentina. Adicionalmente se consultaron los Anuarios de Estadísticas Vitales de Información Básica, las cuales son publicaciones anuales de la misma institución ${ }^{12}$.

\section{Criterios utilizados}

Las causas de mortalidad se agruparon sobre la base de la "Clasificación Estadística Internacional de Enfermedades y Problemas Relacionados con la Salud”, en la décima revisión (CIE-10) ${ }^{13}$. Fueron considerados los códigos CIE-10 desde V00 a Y09. Las causas violentas de mortalidad fueron agrupadas en: "accidentes de transporte" (V00-V99), "otros accidentes" (W00-X59), "suicidio" (X60-X84) y “agresiones" (X85-Y09).

\section{Análisis de la información}

Se calcularon tasas de mortalidad específicas por causas violentas por 100.000 habitantes en población de 10 a 24 años, según agrupamiento de causa y sexo. Se incluyeron para el análisis estadístico los registros cuyos datos estaban completos respecto a edad y sexo.

En el análisis estadístico se compararon las frecuencias mediante análisis del test del $\chi^{2}$, cálculo de Odds Ratio (OR) e intervalos de confianza (IC), y el análisis de variancia (ANOVA) en las variables mensurables.

Posteriormente, la comparación estadística de las tendencias según el grupo de edad y el sexo se llevó a cabo mediante las tasas por trienio 2000-2002 (1T), 2003-2005 (2T) y 2006-2008 (3T). En todos los casos se aceptó un nivel de significación $\mathrm{p}<0,05$ para rechazar la hipótesis nula.

\section{RESULTADOS}

\section{Análisis según las causas violentas agrupadas}

En el grupo estudiado, de 10 a 24 años, el total de defunciones en el año 2000 fueron de 6.778 muertes, de las cuales 4.249 se debieron a causas violentas (el 62,7\%). La tasa de mortalidad para ambos sexos fue de 42,48 muertes por 100.000 habitantes, siendo en los varones de 53,29 muertes por 100.000 y en mujeres de 15,86 por 100.000 habitantes. 
Al final del período analizado (en 2008) se produjeron un total de 7.026 defunciones en el grupo de 10 a 24 años, de las cuales $4.377(62,3 \%)$ fueron debidas a causas violentas. Las tasas de mortalidad por causas violentas por 100.000 habitantes en el año 2008 fueron de 43,09 para ambos sexos, en varones la tasa fue de 51,06 y en mujeres de 17,50, corroborando estos valores la mayor incidencia de muerte por causas violentas en varones al inicio y al final del período analizado ( $\mathrm{p}=0,0015)$, como es visto en la Figura 1. En ambos casos la posibilidad de muerte para los varones fue 17 veces mayor que para las mujeres (OR=17,25; IC95\% 15,30-19,46; $\mathrm{p}<0,0001)$.

\section{Análisis por tipo de causas violentas}

El análisis por tipos de causas violentas en ambos sexos se evidencia en Figura 2, en la cual se presentan las tasas por 100.000 habitantes según "otros accidentes", "accidentes de transporte", "agresiones" y "suicidios". Al analizar según sexo, en todos los casos las tasas observadas en los varones fueron superiores a las registradas en las mujeres, así mismo es de destacar que tanto en varones como en mujeres la tasa de muerte por accidente de transporte se incrementó en 2008 con respecto al 2000 (varones en 2000: $15,14 \%$ o000; varones en $2008: 16,86 \%{ }_{0000}$; mujeres en 2000: 4,68\% $\%_{0000}$; mujeres en 2008: 5,9\% $\%_{0000}$ ).

\section{Análisis según suicidio}

En el grupo de 10 a 24 años en el año 2000 se registraron en Argentina 618 muertes por suicidios, con una tasa de 6,23 cada 100.000 habitantes. De estas muertes, el $77,83 \%$ se produjeron en varones y el $22,17 \%$ en mujeres, siendo sus tasas de 9,58 en varones y 2,80 en mujeres por cada 100.000 habitantes. Es decir que tres de cada cuatro muertes por suicidio se produjeron en los hombres.

En 2008, se registraron 877 muertes por suicidio en el grupo de 10 a 24 años, de las cuales $76,85 \%$ corresponden a

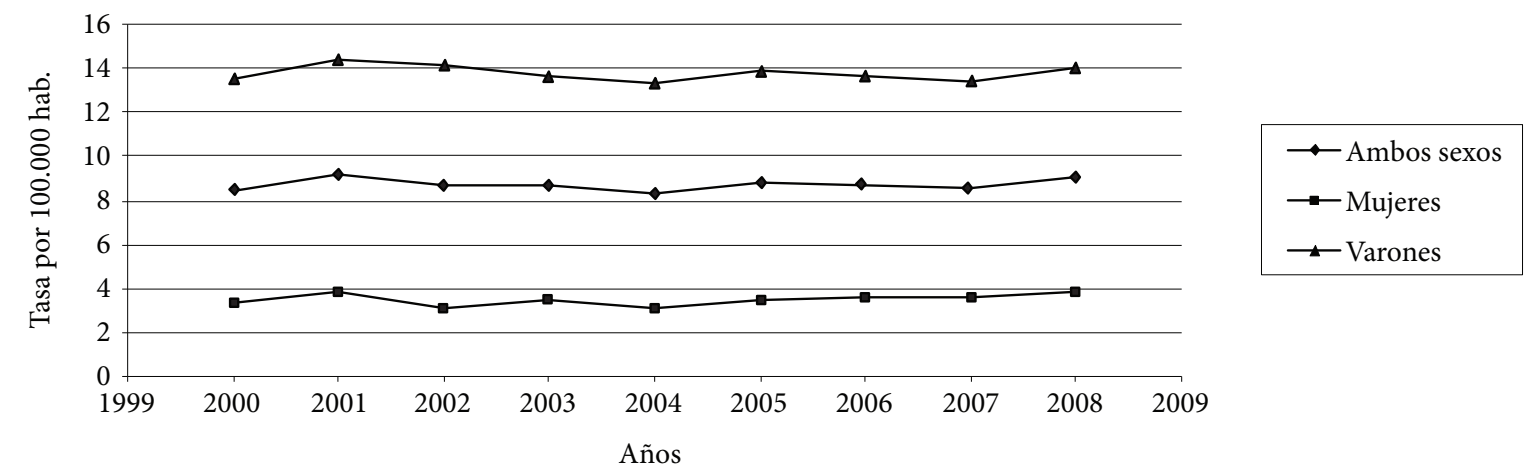

Figura 1. Tasa de mortalidad por causas violentas en el grupo de 10 a 24 años, según sexo. Tasas por 100.000 habitantes, periodo $2000-2008$

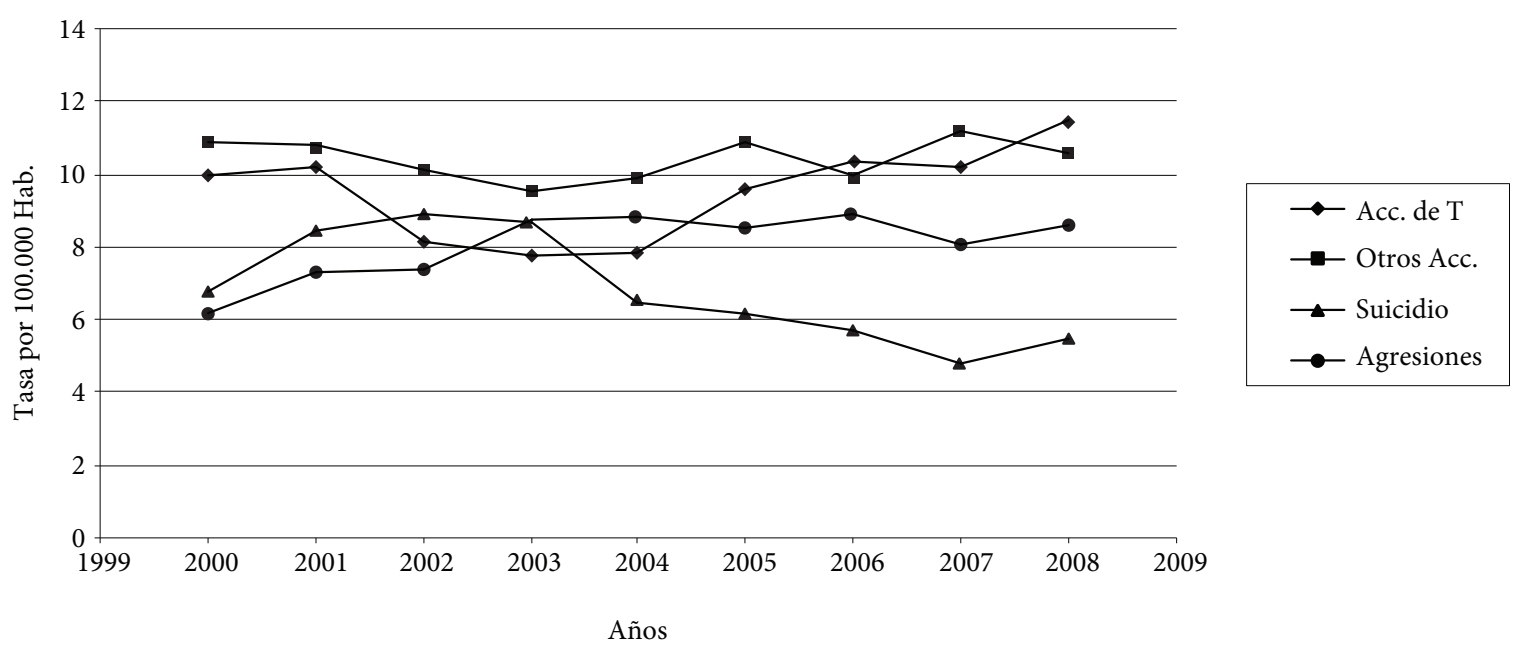

Figura 2. Tasa de mortalidad por causas violentas en el grupo de 10 a 24 años, según grupo de causa, por ambos los sexos. Tasas por 100.000 habitantes, periodo $2000-2008$ 
varones y 23,03\% a mujeres; con una tasa de 8,63 por cada 100.000 habitantes, siendo las tasas de mortalidad específicas de 13,08 para los hombres y 4,04 para las mujeres por cada 100.000 habitantes. Se observó que el incremento de las tasas por suicidio para el grupo de 10 a 24 años del 2000 al 2008 fue del $28 \%$, observándose que la tasa en varones se incrementa en un $27 \%$ y la tasa de mujeres en un $30 \%$.

\section{Análisis de muertes violentas por trienio}

Tal como se observa en la Figura 3, al analizar las causas de muertes violentas por la agrupación de trienios, en el tercero se incrementa significativamente los accidentes de tránsito respecto a los dos anteriores, tanto en mujeres como en varones $(\mathrm{p}<0,01)$. Con respecto a la causa “agresiones” se observó un incremento de las muertes ocurridas por esta en el grupo de 10 a 24 años, tanto en el segundo como en el tercer trienio $\mathrm{y}$ esta tendencia fue mayor en los varones que en las mujeres $(\mathrm{p}<0,01)$. En el caso de "otros accidentes" no se observaron modificaciones en la tasa de mortalidad durante el período analizado. Finalmente, al analizar por trienio las muertes ocasionadas por suicidio en este grupo poblacional se destaca una mayor frecuencia de muertes en el período 2000-2003, la cual se mantiene hasta 2005, este comportamiento fue observado tanto en varones $(\mathrm{p}<0,001)$, como en mujeres $(\mathrm{p}=0,007)$.

\section{DISCUSIÓN}

Los resultados obtenidos muestran que la mortalidad por causas violentas (accidentes de transporte, otros accidentes, suicido y agresiones), en el periodo 2000 a 2008 y en el grupo etario de 10 a 24 años en Argentina, se ha incrementado. Estos resultados acuerdan con lo expresado en los estudios nacionales e internacionales, los cuales refieren que las muertes por causas violentas constituyen el problema de salud pública de mayor importancia para los países latinoamericanos ${ }^{4,9,11,14-17}$. Al efectuar el análisis por tipo de causa se detectó que en Argentina las muertes en jóvenes por accidentes de transportes y agresiones fueron las que presentaron un mayor incremento, destacándose la muerte por suicidio en el período 2000-2005. Estos resultados dan cuenta que la población joven de Argentina, al registrar un incremento de la mortalidad por causas violentas, representando una de las principales causas de muerte en este grupo, refleja un proceso característico de la transición epidemiológica ${ }^{18}$.

Con respecto al comportamiento de la mortalidad por causas violentas y por sexo, se encontró que los varones tienen mayor posibilidad de morir por estas causas que las mujeres y esta tendencia coincide con lo referido en la bibliografía en que el sexo masculino es el más afectado por los comportamientos violentos ${ }^{4,5}$.

En Argentina, en el período 2000-2008, las tasas de suicidio en el grupo etario de 10 a 24 años muestran una tendencia de aumento durante el periodo 2000 a 2003. Esta característica se encuentra relacionada con la crisis socioeconómica que afectó el país en los años 2001 a 2003 y que comprometió aspectos importantes de la salud de las personas entre ellos los aspectos relacionados con la salud mental ${ }^{19}$. Durkehim fue uno de los primeros en demostrar la relación que se presenta entre las variaciones en las tasas de suicidios y los problemas sociales, como crisis económicas y conflictos bélicos ${ }^{20}$. Por otra parte, el incremento de la tasa de suicidio observada en la población joven durante lo período de la crisis económica del país es similar a la informada en la población general por el Ministerio de Salud de la Nación ${ }^{21}$.

Con respecto al análisis de las tasas de suicidio por sexo, se encontró que el grupo de los varones es el más afectado

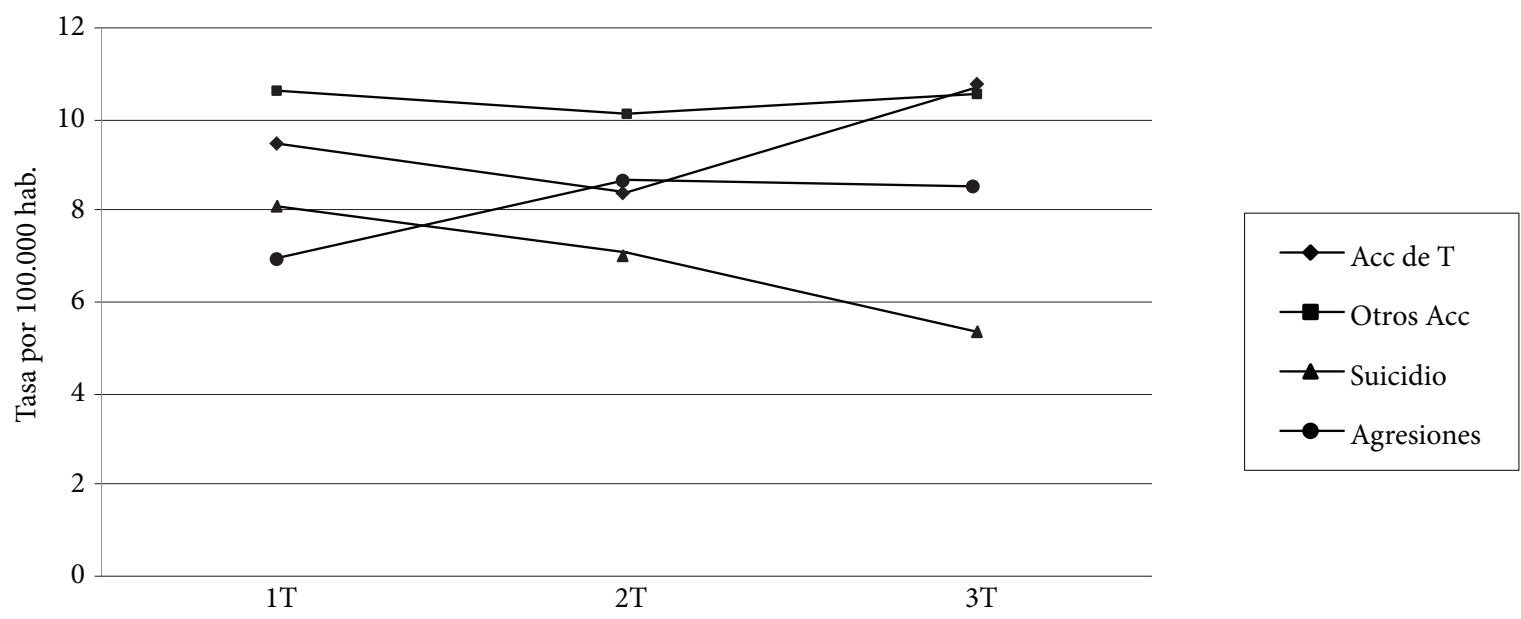

Figura 3. Tasa de mortalidad por causas violentas, según trienio de estudio, en ambos sexos. Tasas por 100.000 habitantes 
y esta tendencia coincide con lo expresado en estudios nacionales e internacionales ${ }^{5,9,11,12,15,16,22}$. Entre las explicaciones que se dan a esta característica se encuentran la alta letalidad de los métodos que eligen para efectuar el suicidio, que son habitualmente el ahorcamiento y las armas de fuego, y las características neurobiológicas y psicopatológicas particulares de cada sexo. Son más frecuentes en los varones las patologías relacionadas al espectro de la impulsividad, como los trastornos de conducta y el uso de sustancias, entre otras ${ }^{17,23-30}$. Asimismo se detectó que aún cuando la tasa de suicidio en varones muestra una tendencia en ascenso, desde el año 2006 se observa que la misma se estaciona y posteriormente disminuye. Entre los factores que podrían explicar esta tendencia, se podría mencionar la paulatina estabilidad económica que adquirió el país durante ese período.

A partir de los resultados se puede concluir que el suicidio en la población de adolescentes y jóvenes en Argentina es un problema que debería abordarse desde la perspectiva de género y desarrollando estudios epidemiológicos para complementar las investigaciones de bases de datos con autopsias psicológicas, a fin de ampliar y profundizar en el conocimiento de los factores que intervienen en esta decisión. Esto permitiría desarrollar medidas sanitarias para intervenir oportunamente, vinculando los aspectos epidemiológicos con las características clínicas, culturales, y socioeconómicas del país.

Teniendo en cuenta que los accidentes de transportes y las agresiones fueron las causas que presentaron un mayor incremento en las tasas de mortalidad estudiadas, y que ambas pueden ser reducidas mediante campañas de educación vial, control de factores de riesgo personales, autocuidado del cuerpo, alerta sobre el consumo de alcohol en los adolescentes y jóvenes, capacitación de los profesionales de primer nivel de atención de salud para la detección y tratamiento precoz de trastornos mentales y del comportamiento en adolescentes y jóvenes ${ }^{31}$, se hace necesario el establecimiento o fortalecimiento de programas de prevención en estas temáticas.

Finalmente, se puede concluir que las muertes por causas violentas constituyen para el país una de las causas de mortalidad de mayor relevancia en la población joven, y teniendo en cuenta que todas son causas de muertes evitables, es imperioso adoptar políticas de promoción de la salud y de prevención enfocadas a disminuir las pérdidas social y económica que generan la muerte de la población joven del país.

\section{REFERENCIAS}

1. Torres Vidal RM, Pernas González A, Martínez Morales M, Peraza Peraza M. Mortalidad por causas externas en el adulto mayor. Cuba. 1970-2004. Rev Temas Estad Salud. 2005;1(2):1-13.

2. Yunes J. Mortalidad por causas violentas en la región de las Américas. Boletín de la Oficina Sanitaria Panamericana. 1993;114:302-16.

3. Yunes J, Rajs D. Tendencia de la Mortalidad por Causas Violentas en la Población General y Entre los Adolescentes y Jóvenes de la Región de las Américas. Cad Saúde Pública. 1994;10 Suppl 1:S88-125.

4. Yunes J, Zubarew T. Mortalidad por causas violentas en adolescentes y Jóvenes un desafío para la Región de las Américas. Braz J Epidemiol. 1999;2(3):45-57.

5. Serfaty E, Foglia L, Masaútis A, Negri G. Mortalidad por causas violentas en adolescentes y jóvenes de 10- 24 años. Argentina 1991- 2000. Rev Vertex. 2007;40:25-30.

6. Duarte C, Bordín I, De Paula C, Hoven C. Conductas suicidas en adolescentes brasileños: análisis exploratorio de prevalencia y factores de riesgo. Rev Psiqu Salud Integral. 2002;2(4):32-7.

7. Vuegen S. Salud de la población adolescente en la Argentina. Rev Arch Argentinos Ped. 2003;101(6):491-4.

8. World Health Organization. Completeness and coverage of death registration data. [cited 2011 June 22]. Available from: http://www.who. int/healthinfo/cod/en/index.html

9. Cardona D, Peláez E, Aidar T, Ribotta B, Alvarez MF. Mortalidad por causas externas en tres ciudades latinoamericanas: Córdoba (Argentina), Campinas (Brasil) y Medellín (Colombia), 1980-2005. Rev Bras Est Pop. 2008;25(2):335-52.
10. Concha-Eastman A, Krug E. Informe mundial sobre la salud y la violencia. Rev Panam Salud Pública. 2002;12(4):227-9.

11. Serfaty E, Andrade JH, Foglia L, Masautis A, Negri G. Suicidio en adolescentes y jóvenes de 10-24 años. Argentina 1991-2000. Rev Sinopsis. 2006;22(42):17-21.

12. Ministerio de Salud de la Nación. Dirección de Estadísticas e información en Salud. [cited 2011 July 1]. Available from: http://www.deis.gov.ar

13. Organización Mundial de la Salud (OMS). Clasificación Internacional de las Enfermedades. Decima Edición. Ginebra: OMS; 1992.

14. Organización Mundial de la Salud (OMS). La salud de los jóvenes: un desafío para la sociedad. Ginebra: OMS; 1986.

15. Basile H. El suicidio adolescente en la Argentina. ALCMEON Rev Arg Clin Neuropsiqu. 2005;12(3):211-31.

16. Cendales R, Vanegas C, Fierro M, Córdoba R, Olarte A. Tendencias del suicidio en Colombia. 1985-2002. Rev Panam Salud Pública. 2007;22(4):231-8.

17. Borges G, Orozco R, Benjet C, Medina-Mora M. Suicidio y conductas suicidas en México: retrospectiva y situación actual. Salud Pública Mex. 2010;52(4):292-304.

18. Anitza Freitez L. Se intensifican los riesgos de morir por causas violentas en la población joven de Venezuela. Rev Venez Análisis Coyuntura. 2008; 14(1):307-35.

19. Zeballos JL. Argentina: efectos socio-sanitarios de la crisis, 2001-2003. Buenos Aires: Organización Panamericana de la Salud; 2003. 
20. Durkheim E. El suicidio. Buenos Aires: Losada; 2004.

21. Ministerio de Salud de la Nación (MSN); Dirección de Promoción de la Salud y Control de Enfermedades No Transmisibles. Perfil epidemiológico del suicidio en la Argentina, 1988-2008. En: Boletín de Vigilancia de Enfermedades no Transmisibles y Factores de Riesgo. Buenos Aires (Argentina): MSN; 2009. p. 58-78.

22. Levi F, La Vecchia C, Lucchini F, Negri E, Saxena S, Maulik PK, et al. Trends in mortality from suicide, 1965-1999. Acta Psichiatr Scand. 2003;108(5):341-9.

23. Llorca Castro F, Ortún Rubio V. Mortalidad innecesariamente prematura y sanitariamente evitable en Costa Rica. Rev Esp Salud Pública. 2010;84(6):771-87.

24. Puentes-Rosas E, López-Nieto L, Martínez-Monroy T. La mortalidad por suicidios: México 1990-2001. Rev Panam Salud Pública. 2004;16(2):102-9.

25. Shaffer D, Gould M, Fisher P. Psychiatry diagnosis in child and adolescent suicide. Arch Gen Psych. 1996;(53):339-48.

26. Dehen KL, Riedner G. Adolescent and young adult mortality by cause. Age gender and country 1955 to 1994. J Adolescent Health. 2002;30(1):29-34.
27. Ocampo R, Bojorquez I, Cortés M. Consumo de sustancias y suicidios en México: resultados del Sistema de Vigilancia Epidemiológica de las Adicciones, 1994-2006. Rev Salud Pública Mex. 2009;51(4): 306-12.

28. Espinoza-Gómez F, Zepeda-Pamplona V, Bautista Hernández V, Hernández-Suarez CM, Newton-Sánchez OA, Plasencia-García GR. Violencia doméstica y riesgo de conducta suicida en universitarios adolescentes. Rev Salud Pública Mex. 2010;52(3):213-9.

29. Gould MS, Greenberg T, Velting D, Shaffer D. Youth suicide risk and preventive interventions: a review of the past 10 years. J Am Acad Child Adolesc Psych. 2003;42(4):386-405.

30. Dervic K, Brent D, Orquendo M. Completed Suicide in Childhood. Psych Clin North Am. 2008;31(2):271-91.

31. Pérez Barrero S, Reytor Sol F. El suicidio y su atención por el médico de la familia. Rev Cubana Med Gen Integr. 1995;11(4):11-8.

Recibido en: 23/05/2012 Aprobado en: 14/08/2012 